\title{
Dual-Rim Sign in the Diagnosis of Cerebral Abscess
}

\author{
Hong Kuan Kok, Hamed Asadi, Mark W. McCusker, Alan O'Hare, John Thornton, Paul Brennan,
} Seamus Looby

Keywords: Infections of the Nervous System, Magnetic Resonance Imaging, Neuroradiology

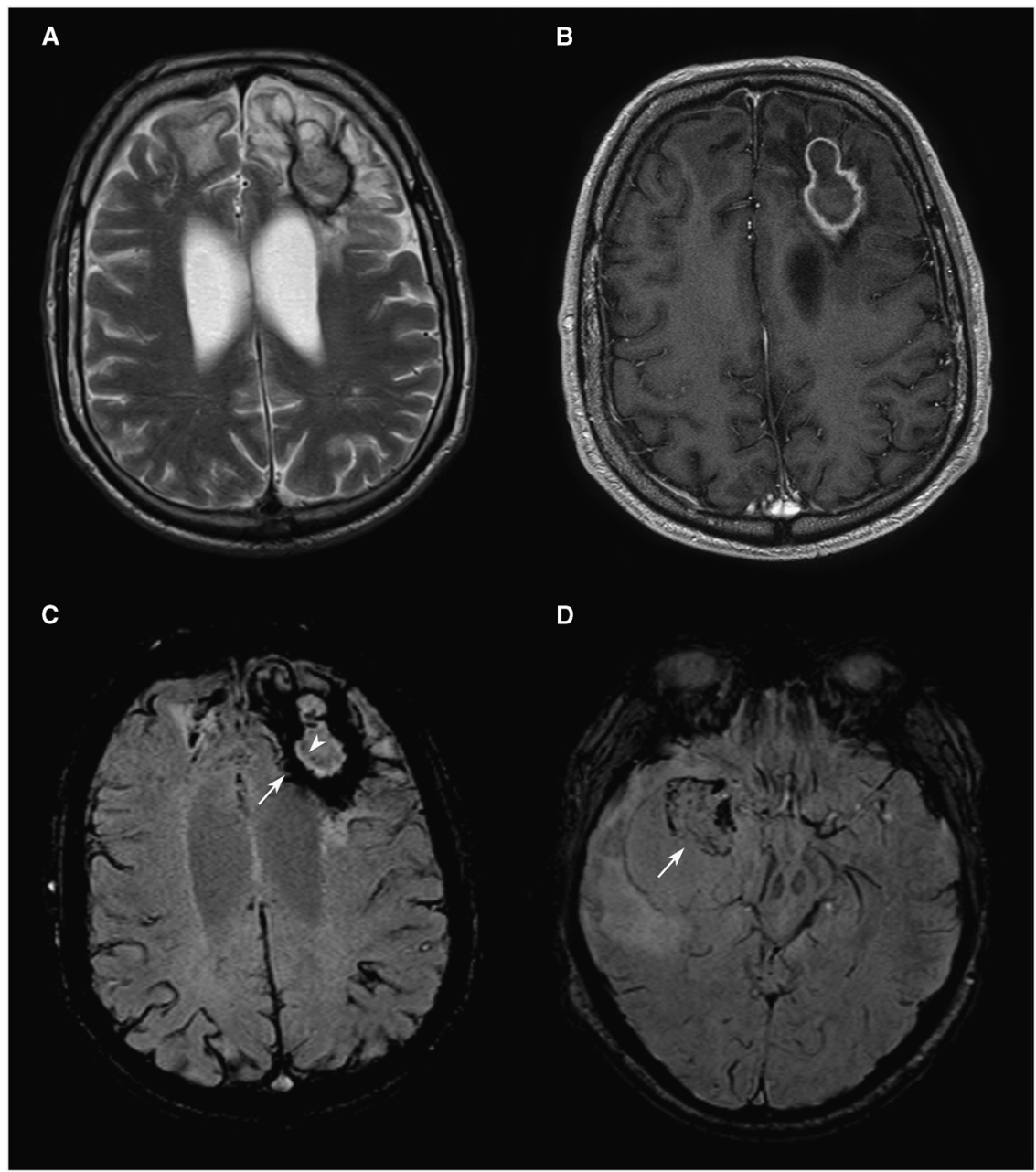

Figure 1: (A) T2-weighted and (B) postcontrast T1-weighted sequences shows a rimenhancing mass in the left frontal lobe with associated vasogenic edema. (C) An SWI sequence demonstrating a dual-rim sign consisting of a complete outer rim of signal hypointensity (arrow) and an inner rim of relative signal hyperintensity compared with the center of the lesion (arrowhead), suggestive of a pyogenic cerebral abscess. (D) An SWI sequence in a different patient with glioblastoma multiforme demonstrating absence of the dual-rim configuration with an incomplete peripheral hypointense rim (arrow). 


\section{Case Presentation}

A 57-year-old man presented with new-onset, generalized tonic-clonic seizures and worsening confusion on a background of recent hospitalization for pelvic fractures sustained in a road traffic accident 3 months previously. His medical history was noncontributory. Clinical examination revealed mild disorientation and confusion but there was no localizing neurologic deficit. Hematological and biochemical parameters were unremarkable. Magnetic resonance imaging of the brain showed a rim-enhancing mass in the left frontal lobe (Figure 1A, B) with associated vasogenic edema and a dual-rim sign on susceptibility-weighted imaging (SWI) sequences (Figure 1C), favoring a diagnosis of pyogenic cerebral abscess. Our patient was treated with intravenous antibiotics and subsequently underwent surgery that confirmed the preoperative diagnosis of a cerebral abscess, which was successfully drained. Blood cultures were positive for Enterobacter species and echocardiography confirmed infective endocarditis of the mitral valve as a source.

Cerebral abscesses are a neurological emergency requiring prompt diagnosis and treatment. ${ }^{1}$ However, many clinical presentations are nonspecific, and the imaging features of pyogenic abscesses, glioblastoma multiforme, and mucinous metastases can overlap. The magnetic resonance imaging features of cerebral abscesses are well-described, typically demonstrating central T2weighted signal hyperintensity, peripheral rim enhancement, and central diffusion restriction on diffusion-weighted imaging sequences. $^{2,3}$ Proton magnetic resonance spectroscopy is also a useful sequence showing lactate, amino acid, acetate, and succinate peaks in abscesses compared with lactate and lipid peaks in tumors. ${ }^{4}$ Despite this, the confident discrimination between cerebral abscesses and necrotic high-grade gliomas can be difficult on imaging studies, particularly where the clinical presentation and diffusion-weighted imaging sequences are equivocal. Another routinely used sequence, SWI, has been described as a relatively specific discriminator for cerebral abscesses by demonstrating a dual-rim sign around the central abscess cavity. ${ }^{5}$ The dual-rim sign consists of a continuous outer rim of signal hypointensity resulting from paramagnetic free radical production by macrophages and an inner rim of relative hyperintensity, postulated to represent granulation tissue in the abscess wall. In contrast, high-grade tumors such as glioblastoma form an incomplete and irregular rim of signal hypointensity on SWI (Figure 1D), which is thought to result from deposition of hemorrhagic products at the tumor periphery.

\section{Disclosures}

The authors do not have any disclosures.

\section{REFERENCES}

1. Osenbach RK, Loftus CM. Diagnosis and management of brain abscess. Neurosurg Clin N Am. 1992;3:403-20.

2. Leuthardt EC, Wippold FJ 2nd, Oswood MC, Rich KM. Diffusionweighted MR imaging in the preoperative assessment of brain abscesses. Surg Neurol. 2002;58:395-402.

3. Rath TJ, Hughes M, Arabi M, Shah GV. Imaging of cerebritis, encephalitis, and brain abscess. Neuroimaging Clin $\mathrm{N}$ Am. 2012;22:585-607.

4. Lai PH, Ho JT, Chen WL, et al. Brain abscess and necrotic brain tumor: discrimination with proton MR spectroscopy and diffusion-weighted imaging. AJNR Am J Neuroradiol. 2002; 23:1369-77.

5. Toh $\mathrm{CH}$, Wei KC, Chang $\mathrm{CN}$, et al. Differentiation of pyogenic brain abscesses from necrotic glioblastomas with use of susceptibility-weighted imaging. AJNR Am J Neuroradiol. 2012;33:1534-8. 